\title{
Comparison between Cardiac Baroreflex Sensitivity Estimates Derived from Sequence and Phase Rectified Signal Averaging Techniques during Head-up Tilt
}

\author{
Beatrice De Maria ${ }^{1,2}$, Vlasta Bari ${ }^{3}$, Giovanni Ranuzzi ${ }^{3}$, Laura Dalla Vecchia $^{1}$, Sergio Cerutti ${ }^{2}$, \\ Alberto Porta ${ }^{3,4}$ \\ ${ }^{1}$ IRCCS Istituti Clinici Scientifici Maugeri, Istituto di Milano, Milan, Italy \\ ${ }^{2}$ Department of Electronics Information and Bioengineering, Politecnico di Milano, Milan, Italy \\ ${ }^{3}$ Department of Cardiothoracic, Vascular Anesthesia and Intensive Care, IRCCS Policlinico San \\ Donato, San Donato Milanese, Milan, Italy \\ ${ }^{4}$ Department of Biomedical Sciences for Health, University of Milan, Milan, Italy
}

\begin{abstract}
The cardiac baroreflex $(c B R)$, the physiological control mechanism that modulates heart rate in response to arterial pressure $(A P)$ changes, is activated by both $A P$ increases and decreases. The sequence method, widely utilized to evaluate $c B R$ sensitivity (cBRS) from spontaneous heart period $(H P)$ and systolic AP (SAP) variations allows the separated computation of $c B R S$ from positive and negative SAP variations. Conversely, the recently proposed bivariate phase rectified signal averaging (PRSA) method was successfully applied in prognostic studies exclusively on positive SAP variations. We extended PRSA method to compute cBRS over negative SAP variations as well and we compared results with those derived from the sequence method in 18 healthy subjects undergoing head-up tilt test with a table inclination of $90^{\circ}$ (HUT). As expected, regardless of the sign of the SAP changes and methods, cBRS moved toward 0 during HUT. The PRSA-based cBRS measure derived from positive SAP changes was extremely correlated with that derived from negative AP variations and the correlation was significantly higher than that obtained from correspondent sequence-based cBRS estimates. In conclusion, the two methods cannot be considered interchangeable in computing cBRS.
\end{abstract}

\section{Introduction}

The cardiac baroreflex (cBR) is a physiological control mechanism that compensates arterial pressure (AP) changes by adjusting heart period (HP). In response to AP increases cBR prolongs HP. Conversely, when AP decreases the cBR shortens HP. As a result of its inner functioning usually cBR is characterized by evaluating the HP responses to both positive and negative AP changes [1].

Historically, the cBR sensitivity (cBRS) measurement was invasively performed by injecting drugs inducing AP changes and observing the HP response [2]. However, in the last thirty years several noninvasive and easy-toperform methods for cBRS estimation have been proposed. These methods are mainly based on the estimation of the cBRS from the spontaneous variability of HP and AP [3]. Among those methods one of the time domain techniques for cBRS estimation, namely the sequence method, allows the separated evaluation of the cBR response to positive and negative systolic AP (SAP) variations [4].

The repertoire of tools for noninvasive assessment of cBRS from spontaneous HP and SAP fluctuations was recently enriched by a tool that demonstrated a remarkable prognostic power in pathological population, namely the bivariate phase rectified signal averaging (PRSA) [5]. Despite PRSA allows the separated estimation of cBRS over both positive and negative SAP variations the method was exclusively utilized over the positive ones [5].

The aim of this study is to extend the usual utilization of the PRSA method to negative SAP changes and to compare cBRS estimates driven by both positive and negative SAP variations with the correspondent estimates obtained from sequence analysis. Comparison was performed on healthy individuals undergoing cBR unloading during orthostatic challenge induced by head-up tilt (HUT) [6].

\section{Experimental protocol and data analysis}

\subsection{Experimental protocol}


Eighteen healthy subjects (age range: 21-48 years, median age $=30$ years, 8 males) were enrolled in the study. A detailed clinical examination excluded subjects with chronic disease, obesity, smoking, alcoholism and chronic drug assumption. The experiments were always performed in the morning, in a quiet and comfortable room. Each subject was studied for 7 minutes at rest in supine position (REST) and for 10 minutes during HUT with tilt table inclination at $90^{\circ}$. Electrocardiogram (ECG) and non-invasive plethysmographic AP (Finometer MIDI, Finapres Medical Systems, Amsterdam, The Netherlands) were continuously recorded at $300 \mathrm{~Hz}$. The study protocol was approved by the "L. Sacco" Hospital Ethics Review Committee and conformed to the principles of the Declaration of Helsinki for medical research involving humans. All subjects provided written informed consent.

\subsection{Beat-to-beat time series extraction}

The HP was defined as the temporal distance between two consecutive R-wave peaks detected on the ECG using a method based on a threshold on the first derivative. R-wave position was fixed by means of parabolic interpolation [7]. The $k$ th SAP was defined as the maximum value of the AP signal within the $k$ th HP. Erroneous HP and SAP detections were detected by visual inspection and corrected. In presence of ectopic beats, a cubic spline interpolation was performed. No more than $5 \%$ of beats were allowed to be manually corrected. Sequences of 256 consecutive beats of HP and SAP series for each experimental condition were selected for further analysis.

\section{3. cBRS estimation via sequence method}

The sequence method for the cBRS estimation is based on the search of sequences of 4 consecutive HP and SAP values characterized by contemporaneous increase (positive sequences) or decrease (negative sequences) of both HP and SAP [4]. The average of the slope of the linear regression in the plane $[\mathrm{SAP}(k), \operatorname{HP}(k+\tau)]$ with $\tau=0$ [8] computed over positive sequences was taken as an estimate of the cBRS and labeled as $\mathrm{cBRS}_{\mathrm{SEQ}+}$. We calculated also the average of the slope of the linear regression in the plane $[\mathrm{SAP}(k), \operatorname{HP}(k+\tau)]$ with $\tau=0$ [8] over negative sequences as an estimate of cBRS labeled as $\mathrm{cBRS}_{\mathrm{SEQ}-}$ [4]. $\mathrm{cBRS}_{\mathrm{SEQ}+}$ and $\mathrm{CBRS}_{\mathrm{SEQ}-}$ were expressed in $\mathrm{ms} / \mathrm{mmHg}$.

\section{4. cBRS estimation via bivariate PRSA method}

The bivariate PRSA method for cBRS estimation was proposed in [5,9]. Briefly, defined as anchor point the cardiac beat $k$ where SAP increased [i.e. $\operatorname{SAP}(k+1)>\operatorname{SAP}(k)$ ], a sequence of 15 consecutive HPs in correspondence to the anchor point were selected. Each HP sequence was composed by the 7 HPs that precede the considered anchor point, the HP at the anchor point and the 7 HPs that follow it. All the identified HP segments are than aligned and centered at the anchor values. Defined $\mathrm{X}(0)$ as the mean of all HPs at the anchors, $\mathrm{X}(-1)$ as the mean of the HPs preceding the anchors, $\mathrm{X}(-2)$ as the mean of the HPs at two beats before the anchors and $\mathrm{X}(1)$ as the mean of the HPs immediately following the anchors, the PRSA-based cBRS driven by positive SAP variations, cBRSPRSA, was calculated as $\mathrm{cBRS}_{\mathrm{PRSA}}=1 / 4 *[\mathrm{X}(0)+\mathrm{X}(1)-\mathrm{X}(-1)-\mathrm{X}(-2)]$. The normalized cBRS PRSA, ncBRS ${ }_{\text {PRSA }}$, was obtained dividing cBRSPRSA by the averaged $\triangle \mathrm{SAP}(k)=\operatorname{SAP}(k+1)-\operatorname{SAP}(k)$ at the anchor [9]. While cBRSPRSA+ was measured in ms, ncBRS PRSA+ $_{\text {was }}$ expressed in the more conventional units for cBRS (i.e. $\mathrm{ms} / \mathrm{mmHg}$ ).

We assessed PRSA-based cBRS by considering as anchor point the time index $k$ where $\mathrm{SAP}(k+1)<\mathrm{SAP}(k)$. The cBRS was computed as described above and the relevant estimate was labeled as cBRS PRSA. Also the normalized cBRS PRSA-, $_{\text {ncBRS }}$ PRSA-, was calculated by normalizing cBRS PRSA- by the averaged $\triangle \mathrm{SAP}(k)=\mathrm{SAP}(k+1)-\mathrm{SAP}(k)$ at the anchor. cBRS PRSAwas expressed in ms, while ncBRSPRSA in $\mathrm{ms} / \mathrm{mmHg}$.

\subsection{Statistical analysis}

The differences between the two experimental conditions (i.e. REST and HUT) were tested by means of paired t-test, or Wilcoxon signed rank test when appropriate. We checked the linear association between the variables within the same method and correspondent

Table 1. Sequence- and PRSA-based cBRSs

\begin{tabular}{|c|c|c|}
\hline cBRS & REST & HUT \\
\hline $\mathrm{cBRS}_{\mathrm{SEQ+}}[\mathrm{ms} / \mathrm{mmHg}]$ & $22.59 \pm 10.9$ & $12.75 \pm 8.03^{*}$ \\
\hline $\mathrm{cBRS}_{\mathrm{SEQ}-}[\mathrm{ms} / \mathrm{mmHg}]$ & $21.0 .8 \pm 7.79$ & $9.67 \pm 5.17 *$ \\
\hline $\mathrm{cBRS}_{\mathrm{PRSA}+}[\mathrm{ms}]$ & $14.45 \pm 6.81$ & $6.73 \pm 4.73 *$ \\
\hline $\mathrm{cBRS}_{\text {PRSA- }}[\mathrm{ms}]$ & $-14.05 \pm 7.23$ & $-6.57 \pm 4.26^{*}$ \\
\hline $\mathrm{ncBRS}_{\text {PRSA+ }}[\mathrm{ms} / \mathrm{mmHg}]$ & $8.65 \pm 5.01$ & $3.34 \pm 2.45^{*}$ \\
\hline ncBRS $S_{\text {PRSA- }}[\mathrm{ms} / \mathrm{mmHg}]$ & $9.04 \pm 5.23$ & $3.77 \pm 2.78^{*}$ \\
\hline
\end{tabular}

REST: resting condition; HUT: head-up tilt at $90^{\circ}$; cBRS: cardiac baroreflex sensitivity; $\mathrm{cBRS}_{\mathrm{SEQ}+}$ : $\mathrm{cBRS}$ calculated by sequence method on positive sequences; cBRS $\mathrm{SEQ}_{\mathrm{SE}}$ : cBRS calculated by sequence method on negative sequences; PRSA: phase rectified signal averaging; BRS $_{\text {PRSA }}$ : cBRS calculated by PRSA method on positive SAP variations; cBRS PRSA-, $_{\text {, }}$ BRS calculated by PRSA method on negative SAP variations; ncBRSPRSA+: normalized cBRS $_{\mathrm{PRSA}+}$; ncBRS ${ }_{\mathrm{PRSA}}$ : normalized

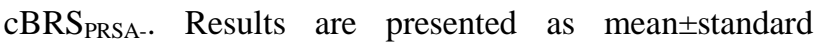
deviation. The symbol $*$ indicates $\mathrm{p}<0.05$ versus REST. 

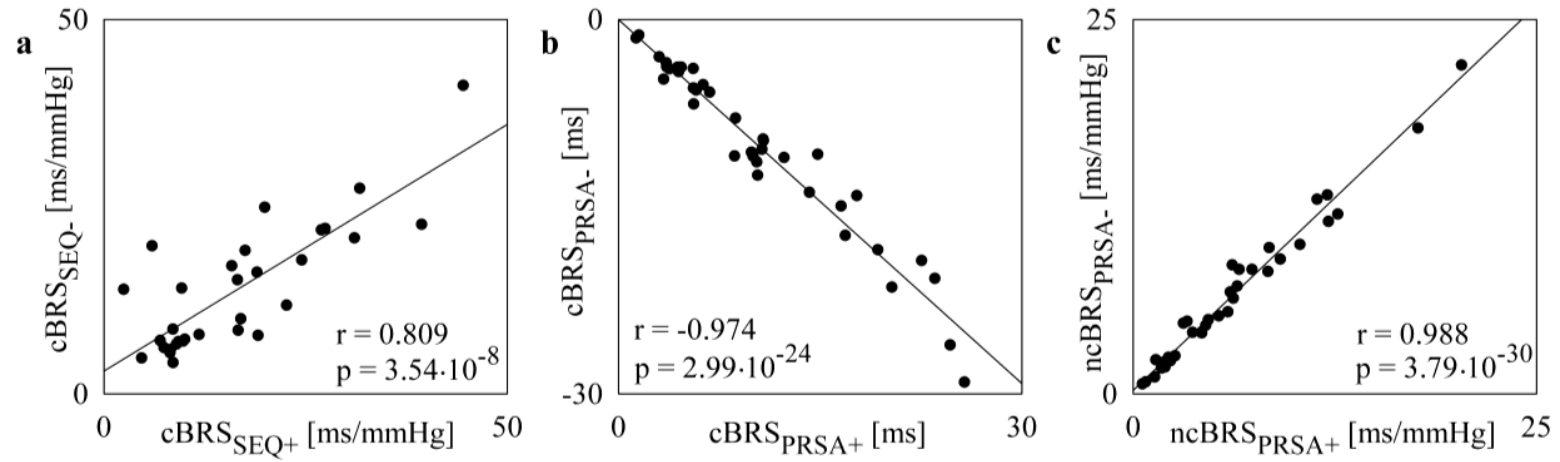

Figure 1. Scatter plots in the plane (cBRS $\left.\mathrm{SEQ}+_{+}, \mathrm{cBRS}_{\mathrm{SEQ}-}\right)(\mathrm{a})$, (cBRS $\mathrm{PRSA}_{+}, \mathrm{cBRS}_{\mathrm{PRSA}}$ ) (b) and (ncBRS $\left.\mathrm{PRSA}_{+}, \mathrm{ncBRS}_{\mathrm{PRSA}-}\right)$ (c). Data are pooled together regardless of the experimental condition. The linear regression line is plotted if $p<0.05$.

variables assessed according to different methods. Pearson product moment correlation coefficient, $r$, was calculated and the null hypothesis of slope equal to 0 was tested after pooling together all the subjects regardless of the experimental condition. A $p<0.05$ was always considered as significant.

\section{Results}

Table 1 shows cBRS indexes estimated by sequence and PRSA methods at REST and during HUT. All the calculated cBRS indexes, i.e. cBRS $_{\mathrm{SEQ}+}$, cBRS $\mathrm{SEQ}_{\mathrm{SEQ}}$,

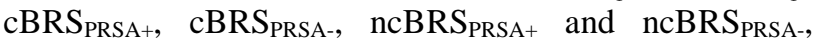
significantly moved toward 0 during HUT.

The results of the correlation analysis between cBRS indexes calculated within the same method are shown in Fig.1. A significant positive correlation was found between cBRS $_{\mathrm{SEQ}+}$ and cBRS $_{\mathrm{SEQ}-}$ (Fig.1a: r=0.809, $\left.\mathrm{p}=3.54 \times 10^{-8}\right)$ and between ncBRS PRSA+ and ncBRS PRSA- $_{\text {- }}$ (Fig.1c: $r=0.988, p=3.79 \times 10^{-30}$ ). A significant negative correlation was found between $\mathrm{cBRS} \mathrm{PRSA}_{+}$and $\mathrm{cBRS} \mathrm{PRSA}_{\mathrm{P}}$ (Fig. 1b: $r=-0.974$ and $\mathrm{p}=2.99 \times 10^{-24}$ ).

The results of the correlation between correspondent cBRS indexes derived from different methods (i.e. sequence or PRSA) are shown in Fig.2. A significant positive correlation was found between $c B R S_{\mathrm{PRSA}}$ and cBRS $_{\mathrm{SEQ}+}$ (Fig.2a: $\mathrm{r}=0.681, \quad \mathrm{p}=1.81 \times 10^{-5}$ ), between

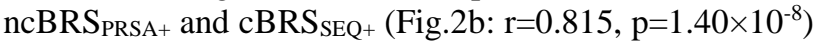
and between ncBRS PRSA- and $\mathrm{cBRS}_{\mathrm{SEQ}}$ (Fig.2d: $\mathrm{r}=0.85$, $\left.\mathrm{p}=2.06 \times 10^{-10}\right)$. A significant negative correlation was detected between cBRS $\mathrm{SEQ}_{\text {- }}$ and cBRS PRSA- (Fig.2c: $\left.\mathrm{r}=-0.78, \mathrm{p}=5.45 \times 10^{-8}\right)$.

\section{Discussion}

The study applied sequence and bivariate PRSA methods for the cBRS estimation to a standard protocol evoking $\mathrm{cBR}$ unloading via a postural challenge. The originality of the study lies in the direct comparison between cBRS estimates computed over positive and negative SAP variations.
It is well-known that reflex HP changes induced by AP increase or decrease are asymmetric and this information is physiological relevant [1]. For example, the response to SAP positive changes seems to be stronger that that caused by the negative ones [10]. This asymmetry can be explored using sequence method by differentiating positive and negative SAP variations [4]. Also the recently developed method based on bivariate PRSA [5,9] can provide separated identification of the HP variation according to the sign of SAP changes. Despite this possibility the PRSA-based cBRS was computed exclusively for positive SAP changes [5,9]. In this study cBRS was estimated based on sequence and PRSA methods by separately exploring the HP response to positive and negative SAP variations. We found that all the cBRS indexes moved toward 0 during HUT, as physiologically expected due to the sympathetic activation and vagal withdrawal caused by the orthostatic stressor [11-13]. This modification was observed
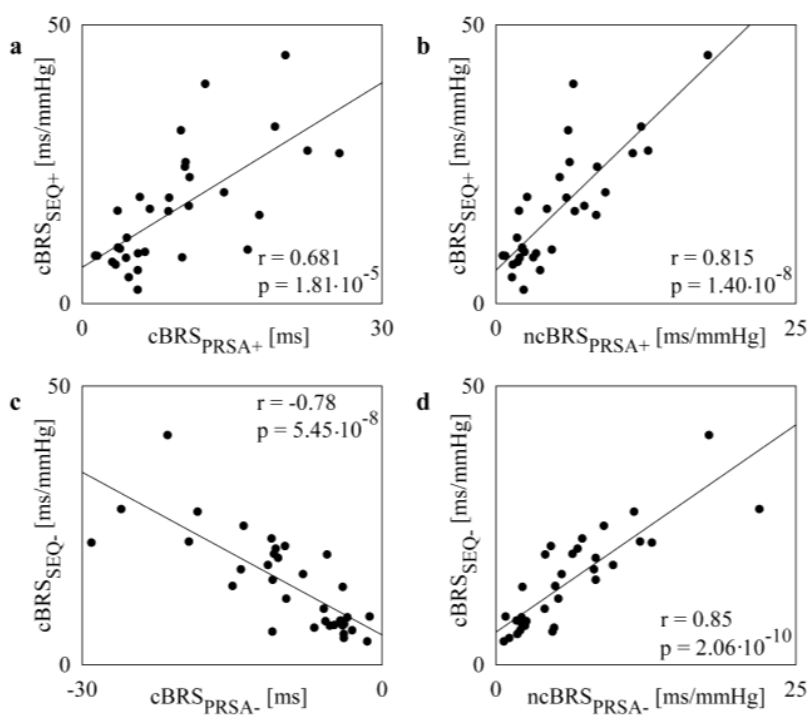

Figure 2. Scatter plot in the plane (cBRS $\left.\mathrm{PRSA}_{+}, \mathrm{cBRS}_{\mathrm{SEQ}_{+}}\right)$ (a), (ncBRS PRSA+, $\left.\mathrm{cBRS}_{\mathrm{SEQ}+}\right)$ (b), (cBRS $\left.\mathrm{PRSA}, \mathrm{cBRS}_{\mathrm{SEQ}-}\right)$ (c), and (ncBRS PRSA-, $\left.\mathrm{cBRS}_{\mathrm{SEQ}-}\right)$ (d). Data are pooled together regardless of the experimental condition. The linear regression line is plotted if $p<0.05$. 
independently of the sign of the SAP variation, thus suggesting that the stimulus is strong enough to overcome possible asymmetric behavior of the cBR control in healthy subjects.

The analysis of the correlation between the measurements within the same method underlined differences between the two techniques. Indeed, the degree of association between $\mathrm{cBRS}_{\mathrm{SEQ}+}$ and $\mathrm{cBRS}_{\mathrm{SEQ}-}$ was significantly smaller than that between cBRS $\mathrm{PRSA}_{+}$ and CBRS PRSA.. This result suggests a certain degree of stiffness of the PRSA method in assessing the asymmetric behavior of cBR. This stiffness might have played a relevant role in improving the repeatability and robustness of the PRSA-based cBRS markers in pathological population [14]. However, this stiffness might limit their application in healthy subjects when a key feature such as cBR asymmetry is under scrutiny. This consideration is supported further by the fact that the correlation between corresponding indexes computed by the two methods, even though significant, was not very strong. We also confirmed previous observations [9] that the PRSA-based cBRS indexes calculated by normalizing by the SAP variation did not differ substantially from those without normalization, thus stressing again a certain degree of independency of PRSA-based cBRS markers to SAP changes.

\section{Conclusions}

The cBRS was calculated using sequence and PRSA methods during postural challenge in healthy subjects. Despite cBRSs computed over positive and negative SAP changes were significantly correlated regardless of the method, the lack of variability about the linear trend when indexes are computed by the PRSA method suggests its greater stiffness compared to sequence analysis. This stiffness, likely to have contributed to PRSA method success in pathological populations [14], might hide complex physiological patterns (i.e. different responses to positive or negative SAP changes) in healthy subjects.

\section{References}

[1] T. G. Pickering, B. Gribbin and P. Sleight, "Comparison of the reflex heart rate response to rising and falling arterial pressure in man," Cardiovasc. Res., vol. 6, pp. 277-283, 1972.

[2] M. T. La Rovere, G. D. Pinna and G. Raczak, "Baroreflex sensitivity: measurement and clinical implications," Ann. Noninvasive Electrocardiol., vol. 13, pp. 191-207, 2008.

[3] A. Porta, V. Bari, T. Bassani, A. Marchi, V. Pistuddi and M. Ranucci, "Model-based causal closed loop approach to the estimate of baroreflex sensitivity during propofol anesthesia in patients undergoing coronary artery bypass graft," J. Appl. Physiol., vol. 115, pp. 1032-1042, 2013.

[4] G. Bertinieri, M. di Rienzo, A. Cavallazzi, A.U. Ferrari, A. Pedotti and G. Mancia, "A new approach to analysis of the arterial baroreflex," J. Hypertens., vol. 3, pp. S79-S81, 1985.

[5] A. Bauer, A. Morley-Davies, P. Barthel, A. Muller, K. Ulm, M. Malik and G. Schmidt, "Bivariate phase-rectified signal averaging for assessment of spontaneous baroreflex sensitivity: pilot study of the technology," J. Electrocardiol., vol. 43, pp. 649-653, 2010.

[6] A. Porta, A. M. Catai, A. C. Takahashi, V. Magagnin, T. Bassani, E. Tobaldini, P. van de Borne and N. Montano, "Causal relationships between heart period and systolic arterial pressure during graded head-up tilt," Am. J. Physiol., vol. 300, pp. R378-R386, 2011.

[7] A. Porta, G. Baselli, F. Lomnbardi, S. Cerutti, R. Antolini, M. Del Greco, F. Ravelli and Nollo G., "Performance assessment of standard algorithms for dynamic R-T interval measurement: comparison between R-Tapex and R-Tend approach," Med. Biol. Eng. Comput., vol. 36, pp. 35-42, 1998.

[8] A. Porta, G. Baselli, O. Rimoldi, A. Malliani and M. Pagani, "Assessing baroreflex gain from spontaneous variability in conscious dogs: role of causality and respiration," Am. J. Physiol., vol. 279, pp. H2558-H2567, 2000.

[9] A. Muller, A. Morley-Davies, P. Barthel, K. Hnatkova, A. Bauer, K. Ulm, M. Malik and G. Schmidt, "Bivariate phase-rectified signal averaging for assessment of spontaneous baroreflex sensitivity: normalization of the results," J. Electrocardiol., vol. 45, pp. 77-81, 2012.

[10] J. Parlow, J. P. Viale, G. Annat, R. Hughson and L. Quintin, "Spontaneous cardiac baroreflex in humans. Comparison with drug-induced responses," Hypertension, vol. 25, pp. 1058-1068, 1995.

[11] W.H. Cooke, J.B. Hoag, A.A. Crossman, T.A. Kuusela, K.U.O. Tahvanainen and D.L. Eckberg, "Human responses to upright tilt: a window on central autonomic integration," J. Physiol., vol. 517, pp. 617-628, 1999.

[12] R. Furlan, A. Porta, F. Costa, J. Tank, L. Baker, R. Schiavi, D. Robertson, A. Malliani and R. Mosqueda-Garcia, "Oscillatory patterns in sympathetic neural discharge and cardiovascular variables during orthostatic stimulus," Circulation, vol. 101, pp. 886-892, 2000.

[13] A. Marchi, V. Bari, B. De Maria, M. Esler, E. Lambert, M. Baumert and A. Porta, "Simultaneous characterization of sympathetic and cardiac arms of the baroreflex through sequence techniques during incremental head-up tilt," Front. Physiol., vol. 7, pp. 438, 2016.

[14] G.D. Pinna, A. Porta, R. Maestri, B. De Maria, L.A. Dalla Vecchia and M.T. La Rovere, "Different estimation methods of spontaneous baroreflex sensitivity have different predictive value in heart failure patients," J. Hypertens. 2017 (in press).

Address for correspondence:

Beatrice De Maria, PhD Candidate - Politecnico di Milano, Department of Electronics Information and Bioengineering IRCCS Istituti Clinici Scientifici Maugeri, Via Camaldoli 64, 20138 Milano, Italy

Tel: +390250725286

Email: beatrice.demaria@icsmaugeri.it 